\title{
Rationality in Conformal Field Theory
}

\author{
Greg Anderson ${ }^{1}$ and Greg Moore ${ }^{2}$ \\ ${ }^{1}$ School of Mathematics, Institute for Advanced Study, Princeton, NJ 08540, USA \\ ${ }^{2}$ School of Natural Sciences, Institute for Advanced Study, Princeton, NJ 08540 USA
}

\begin{abstract}
We show that if the one-loop partition function of a modular invariant conformal field theory can be expressed as a finite sum of holomorphically factorized terms then $c$ and all values of $h$ are rational.
\end{abstract}

\section{Introduction}

There is some evidence for an underlying arithmetic nature of conformal field theory. If this is so then the fundamental data of the theory might be expected to have an arithmetic significance. These data include the central extension $c$ and highest weights $\left\{h_{i}\right\}$ of representations of the Virasoro algebra, together with operator product expansion coefficients [1]. While the arithmetic nature of these data is still almost wholly conjectural, it is interesting to note that in all known conformal field theories the value of $c$ is rational. In this note we prove that in a certain subclass of conformal theories the values of $c$ (and of $h_{i}$ ) must be rational.

The class of conformal theories we will discuss are known as rational conformal field theories. Conformal field theories are a distinguished class of two-dimensional quantum field theories that are partially characterized by the requirement that the Hilbert space of the theory $H$ be a representation of a product of commuting Virasoro algebras: Vir $\oplus \overline{\operatorname{Vir}}$ of the form

$$
H=\oplus_{a, b \geqq 0} V\left(h_{a}, c\right) \otimes \bar{V}\left(\bar{h}_{b}, c\right),
$$

where $V(h, c)$ is the irreducible highest weight representation characterized by the central extension $c$ (the same for all representations in (1.1)) and highest weight $h$, i.e. $L_{0} v=h v$ for the highest weight vector $v$. In conformal field theory $h_{a} \geqq 0$, and the degeneracy of the states with $h=\bar{h}=0$ is exactly one. Another distinguishing characteristic of conformal field theory is modular invariance, which, among other things states that the one loop partition function:

$$
Z(\tau)=\operatorname{Tr} q^{L_{0}-c / 24} \bar{q}^{\bar{L}_{0}-c / 24}=\sum_{a, b \geqq 0} N_{a b} \chi\left(h_{a}, c\right) \bar{\chi}\left(\bar{h}_{b}, c\right)
$$

is modular invariant. Here $N_{a b}$ is the degeneracy of representations $\left(h_{a}, \bar{h}_{b}\right), \chi(h, c)$ is the character of the representation $V(h, c)$, and $q=e^{2 \pi i \tau}$, where $\tau \in \mathscr{H}$, the upper 
half plane. These are other properties a field theory must satisfy to be a conformal field theory, but we will not use these in this paper.

Conformal field theories are very special objects, yet the space of these objects is not well known. One might hope that the imposition of various finiteness conditions can lead to a more manageable class of theories. An example of this is Cardy's celebrated result that if the number of primary field is finite then $c<1$ [2]; conformal field theories with $c<1$ have indeed been classified. As part of an attempt to classify all conformal field theories D. Friedan and S. Shenker [3] have suggested that an interesting class of theories are those for which the $\mathbf{Z}^{+} \times \mathbf{Z}^{+}$ matrix $N_{a b}$ has finite rank. These theories are known as rational conformal field theories.

Let us analyze more closely the condition of finiteness of the rank. By rearranging rows and columns we can require that if $N$ has rank $k$ then

$$
\left.\operatorname{det} N_{a b}\right|_{1 \leqq a, b \leqq k} \neq 0 \text {. }
$$

Thus viewing the columns as vectors we can take the first $k$ columns as a basis for the image of $N$. Hence, for $j>k$,

$$
N_{a j}=\sum_{i=1}^{k} D_{j i} N_{a i} \quad a=1,2, \ldots
$$

The matrix $D_{j i}$ is rational by Kramers' rule. It follows that the sum (1.2) can be rewritten as

where

$$
\sum_{i=1}^{k} f_{i} \bar{g}_{i}
$$

$$
\begin{aligned}
& f_{i}=\sum_{a \geqq 1} N_{a i} \chi\left(h_{a}, c\right) \equiv \sum_{a} d_{a, i} q^{h_{a, i}} \\
& \bar{g}_{i}=\bar{\chi}\left(\bar{h}_{i}, c\right)+\sum_{j>k} D_{j i} \bar{\chi}\left(\bar{h}_{j}, c\right) \equiv \sum_{a} e_{a, i} \bar{q}^{\bar{h}_{a, i}}
\end{aligned}
$$

are holomorphic and antiholomorphic functions with $q$-Puiseux expansions having rational coefficients. (We will distinguish $q$-expansions, which have integral powers of $q$ from $q$-Puiseux expansions, for which the powers of $q$ are not necessarily integral. $)^{1}$

In this note we prove the

Theorem. The constraint of modular invariance together with the rationality of $d_{a, i}, e_{a, i}$ implies that the powers of $q$ in (1.4) are rational.

The essential step of the proof is Proposition 2 below, which is a purely arithmetic statement. To apply this to conformal field theory note that since the vacuum has $h=0, c$ and all conformal weights are rational.

The rationality of the coefficients $d_{a, i}$ and $e_{a, i}$ is sufficient for our purposes, but we note that the truth of a conjecture of E. Martinec [5] would imply that the

\footnotetext{
${ }^{1}$ Comments and conjectures on the nature of theories having a partition function of the form (1.3) were also made in [4]
} 
coefficients of $f_{i}, \bar{g}_{i}$ can be taken to be integers. The coefficients of $f_{i}$ are manifestly integral. Since the denominators of $D_{j i}$ are no larger than det $N_{a b}$ we can enlarge the set of vectors $N_{a i}$ to a set with $1 \leqq i \leqq K$ for $K \geqq k$ which generate the image of $N$ as a Z $\mathbf{Z}$-module. For this larger set the new matrix $D_{j i}$ and hence the corresponding coefficients $e_{a, i}$ are integers. ${ }^{2}$

Some examples of known rational conformal field theories are:

1. Rational toroidal compactifications and orbifolds $[3,4,6$,$] . For these c=d$, the dimension of the torus.

2. The unitary discrete series [7], with

$$
c=1-\frac{6}{m(m+1)} \quad m=2,3, \ldots .
$$

3. $\mathbf{N} / N \mathbf{Z}$ parafermion algebras $[8,9]$, with

$$
c=\frac{2(N-1)}{N+2} \quad N=2,3 \ldots .
$$

4. The new discrete series of $[5,10]$ with

$$
c=\frac{\left(n^{2}-1\right) M N(M+N+2 n)}{(M+N+n)(M+n)(N+n)}
$$

with $n \geqq 2 M, N \geqq 1$.

5. The WZW model [11] for a group $G$ at level $k$ with

$$
c=\frac{k \operatorname{dim} G}{c_{v}+k},
$$

where $c_{v}$ is the Casimir of the adjoint representation (the dual Coxeter number).

6. Isolated $c=1$ models [12].

7. Tensor products of the above.

To begin our proof, we note that the modular invariance of (1.3) implies that $f_{i}, \bar{g}_{i}$ form finite dimensional representations of the modular group $\Gamma$. Hence we are naturally led to consider functions $f(\tau)$ which are holomorphic in $\mathscr{H}$, have an expansion in (not necessarily integral) powers of $q$, and for which the span over C: $\operatorname{Span}\{f \circ \gamma\}_{\gamma \in \Gamma}$ is finite dimensional. In order to work effectively with these functions we first must make a mathematical digression.

\section{Some Mathematical Tools}

A.Differential Equations. In [13] D. Friedan and S. Shenker proposed an approach to classifying conformal field theories based on the geometry of flat vector bundles over moduli space. In the case of finite dimensional vector bundles, such structures have been well studied in the mathematics literature [14-16]. We will draw on

\footnotetext{
${ }^{2}$ We thank D. Friedan for a useful discussion about this
} 
some of the classical results of the subject in our proof. Therefore, we summarize here the relevant results.

We begin with a well-known result:

Lemma 1. Let $y_{1}, \ldots, y_{n}$ be linear independent meromorphic functions in a connected open subset $U \subset \mathbf{C}$, and let $V$ be their span over $\mathbf{C}$. Let $E=e(d / d x)$ be a meromorphic vector field on $U$ not identically zero. Then there exist unique meromorphic functions $k_{1}, \ldots, k_{n}$ on $U$ such that the space of solutions of

$$
E^{n} y+k_{1} E^{n-1} y+\cdots k_{n} y=0
$$

is precisely $V$.

Proof. Given the $y_{i}$ we can construct the $k_{i}$ as follows. Any vector $y$ in $V$ can be written $y=\Sigma c_{i} y_{i}$. Therefore:

$$
\operatorname{det}\left(\begin{array}{cccc}
y & y_{1} & \cdots & y_{n} \\
E y & E y_{1} & \cdots & E y_{n} \\
\cdot & \cdot & \cdots & \cdot \\
E^{n} y & E^{n} y_{1} & \cdots & E^{n} y_{n}
\end{array}\right)=0 .
$$

On the other hand, by linear independence the $y_{i}$ have a Wronskian which is not identically zero, so that, expanding the above equation and dividing by the Wronskian we arrive at Eq. (2.1), with

$$
k_{i}=\frac{\operatorname{det}\left(\begin{array}{ccc}
y_{1} & \cdots & y_{n} \\
\cdot & \cdots & \cdot \\
E^{i-2} y_{1} & \cdots & E^{i-2} y_{n} \\
E^{i} y_{1} & \cdots & E^{i} y_{n} \\
\cdot & \cdots & \cdot \\
E^{n} y_{1} & \cdots & E^{n} y_{n}
\end{array}\right)}{\operatorname{det}\left(\begin{array}{ccc}
y_{1} & \cdots & y_{n} \\
E_{1} & \cdots & E^{\prime} y_{n} \\
\cdot & \cdots & \cdot \\
E^{n} y_{1} & \cdots & E^{n} y_{n}
\end{array}\right)} .
$$

If we apply Lemma 1 with $U=\mathscr{H}$, and $E=q(d / d q)=(1 / 2 \pi i)(d / d \tau)$, then it follows that the following two conditions are equivalent:

(I) The coefficients $k_{1}, \ldots, k_{n}$ of $(2.1)$ are invariant under $T: \tau \rightarrow \tau+1: \mathscr{H} \rightarrow \mathscr{H}$.

(II) $\operatorname{Span}\left\{y_{1}, \ldots, y_{n}\right\}=\operatorname{Span}\left\{y_{1} \circ T, \ldots, y_{n} \circ T\right\}$.

The local analysis of singular points of differential equations proceeds by passing from a neighborhood of a singular point, which is a punctured disk, to its universal cover, the upper half plane. Thus it is natural to consider the following growth condition:

Definition. A holomorphic function $f$ on $\mathscr{H}$ is of moderate growth in vertical 
strips if for all real numbers $a<b$ and $C>0$ there exist real numbers $A>0$ and $B>0$ such that

$$
|f(\tau)| \leqq A e^{B \operatorname{Im} \tau} \quad a \leqq \operatorname{Re}(\tau) \leqq b, \quad \operatorname{Im} \tau \geqq C .
$$

We can now summarize the main results from the theory of regular singular points. A singular point of the differential equation (2.1) is said to be a regular singular point if, denoting by $m$ the order of vanishing of the vector field $E$ at the singular point, the order of vanishing of $k_{i}$ is not less than $(m-1) i$. Equivalently, if we use a local parameter $x$ near the point and take $E=x(d / d x)$ then the $k_{i}$ are required to be holomorphic at $x=0$. If $y_{1}, \ldots, y_{n}$ are $\mathrm{C}$-linearly independent holomorphic functions on $\mathscr{H}$ satisfying the conditions (I) (hence (II)), then the following are equivalent:

(A) $y_{1}, \ldots, y_{n}$ are of moderate growth in vertical strips.

(B) Each $y_{i}$ is a finite linear combination of functions of the form $q^{\theta} \tau^{k} g(q)$, where $\theta \in \mathbf{C}, k \in \mathbf{Z}, k \geqq 0, g$ is holomorphic on the open unit disk.

(C) The functions $k_{i}$ in (2.1) have a $q$ expansion (i.e. an expansion in integral powers of $q$ ) for $\operatorname{Im}(\tau) \gg 0$, and the $q$-expansion of each coefficient $k_{i}$ of (2.1) contains no negative powers of $q$.

We briefly explain why this is reasonable. The equivalence of (A) and (B) is a simple consequence of the properties of removable singularities. The equivalence of $(A)$ and $(C)$ is fairly elementary if the number $k$ in $(B)$ is zero (i.e. if there is only semisimple monodromy). In this case, for $(C) \Rightarrow(A)$ one can substitute power series expansions and require that they satisfy the differential equation (the method of Frobenius), while for $(\mathrm{A}) \Rightarrow(\mathrm{C})$ one simply substitutes into (2.2). For a careful discussion see [16] and [15], especially Sect. II.1.

We can apply these results to the class of functions described in the introduction. More precisely we make the following definition:

Definition. A holomorphic function $f$ on $\mathscr{H}$ is quasi-automorphic if the following hold:

1. $\operatorname{Span}\{f \circ \gamma\}_{\gamma \in \Gamma}$ is finite dimensional.

2. $f \circ \gamma$ is of moderate growth in vertical strips for all $\gamma \in \Gamma$.

In order to avoid complications of ramification points we consider the subgroup $\Gamma(2)$ of Mobius transformations defined by $S L(2, \mathbf{Z})$ matrices

$$
\left(\begin{array}{ll}
a & b \\
c & d
\end{array}\right) \equiv\left(\begin{array}{ll}
1 & 0 \\
0 & 1
\end{array}\right) \bmod 2
$$

We define the $\Gamma(2)$ modular function

$$
\lambda=\frac{\vartheta_{2}^{4}}{\vartheta_{3}^{4}}: \mathscr{H} / \Gamma(2) \stackrel{\sim}{\longrightarrow} P^{1}-\{0,1, \infty\} .
$$

$\lambda$ is known as Picard's $\lambda$-function. A crucial property satisfied by $\lambda$ is that its $q$ expansion contains only half-integer powers of $q$, and has integer coefficients. 
Explicitly:

$$
\lambda=16 q^{1 / 2} \prod_{n}\left(\frac{1+q^{n}}{1+q^{n-1 / 2}}\right)^{8} .
$$

We now apply once more Lemma 1 with $U=\mathscr{H}$ and $E=d / d \lambda=(d \lambda / d \tau)^{-1} d / d \tau$ to see that the following conditions are equivalent:

(I') The coefficients $k_{1}, \ldots, k_{n}$ in (2.1) are $\Gamma(2)$ invariant.

(II') $\operatorname{Span}\left\{y_{1}, \ldots, y_{n}\right\}=\operatorname{Span}\left\{y_{1} \circ \gamma, \ldots, y_{n} \circ \gamma\right\}$ for all $\gamma \in \Gamma(2)$.

Applying the theory of regular singular points in this case we have

Proposition 1. Let $y_{1}, \ldots, y_{n}$ be $\mathbf{C}$-linearly independent holomorphic functions on $\mathscr{H}$, and let $E=d / d \lambda$. If the $y_{i}$ satisfy either (hence both) of the conditions ( $\left.\mathrm{I}^{\prime}\right),\left(\mathrm{II}^{\prime}\right)$ then the following are equivalent:

$\left(\mathrm{A}^{\prime}\right)$ All singular points of $(2.1)$ on the $\lambda$-plane (including $\left.\lambda=0,1, \infty\right)$ are regular. At each singular point $\lambda_{0} \neq 0,1, \infty,(2.1)$ has $n$ linearly independent solutions in the form of power series (with integral powers) in $\lambda-\lambda_{0}$. In particular, $k_{i}$ are rational functions of $\lambda$.

(B') $y_{1}, \ldots, y_{n}$ are quasi-automorphic.

We make two remarks. The first remark is that the above theory has a beautiful interpretation in terms of flat vector bundles over moduli space. We refer to $[15,16]$ for a discussion of this.

The second remark is that differential equations for the partition function of the discrete series are known to follow from the existence of null vectors in the Virasoro algebra $[1,17,18]$. On the other hand, from Proposition 1 we see that similar differential equations are a direct consequence of the finite rank of $N_{a b}$, even when $c>1$. It is natural to conjecture that these differential equations also follow from a null vector in the larger algebra whose existence is conjectured in [5].

We now describe our second tool.

B. Algebraic Automorphisms. An algebraic automorphism of the field $\mathbf{C}$ is a bijective map $\varphi: \mathbf{C} \rightarrow \mathbf{C}$ which preserves addition and multiplication. Note that $\varphi(1)=1$, hence $\varphi(m)=m$ for any integer $m$, hence $\varphi(m / n)=m / n$, i.e., $\varphi$ fixes $\mathbf{Q}$. Note further that $\varphi$ is not required to be continuous. We will use the following standard fact about such automorphisms:

Lemma 2. If $\theta$ is irrational then there is an algebraic automorphism $\varphi$ of $\mathbf{C}$ such that $\varphi(\theta) \neq \theta$.

Although this fact is a standard part of the folklore of number theory, we will indicate why it is true. ${ }^{3}$ A simple example of the kind of transformation we have in mind is $\varphi: a+b \sqrt{2} \rightarrow a-b \sqrt{2}$ when $a, b \in \mathbf{Q}$, which must then be extended to the entire complex numbers. To show this we assume some familiarity with Galois theory $[19,20]$. To begin with, suppose $\theta$ is an algebraic number. Denote by $\overline{\mathbf{Q}}$

\footnotetext{
${ }^{3}$ Actually all we will need is an automorphism of a finitely generated extension of $\mathbf{Q}$, but it is not much harder to prove the more general statement
} 
the field of algebraic numbers, which can be decomposed in a tower of Galois extensions

$$
\overline{\mathbf{Q}}=\bigcup_{i} K_{i}
$$

with $K_{i} \subset K_{i+1}$ and $K_{i} / \mathbf{Q}$ Galois. Suppose $\theta \in K_{i}$. Then there is an element $\varphi$ of $\operatorname{Gal}\left(K_{i} / \mathbf{Q}\right)$ for which $\varphi(\theta) \neq \theta$, and, by the fundamental theorem of Galois theory we can lift $\varphi$ to all the higher $K_{j}$, hence to $\operatorname{Aut}(\overline{\mathbf{Q}})$. To get to $\operatorname{Aut}(\mathbf{C})$ we choose (using the axiom of choice!) a transcendence basis $S$ for $\mathbf{C}$ over $\overline{\mathbf{Q}}$, i.e. a maximal algebraically independent set of complex numbers over $\overline{\mathbf{Q}}$. By requiring that $\varphi$ fix this basis we obtain an element of $\operatorname{Aut}(\overline{\mathbf{Q}}(S))$. By the definition of a transcendence basis, $\mathbf{C}$ is algebraic over $\mathbf{Q}(S)$ so by using once again Galois theory and (transfinite) induction we can extend $\varphi$ to $\operatorname{Aut}(\mathbf{C})$. Similarly, if $\theta$ is transcendental we can complete it to a transcendence basis $S$ for $\mathbf{C}$ over $\mathbf{Q}$ and define $\varphi(\theta)=-\theta$, while $\varphi$ fixes the remaining elements of $S$. As before we can extend $\varphi$ to $\operatorname{Aut}(\mathbf{C})$.

\section{Rationality of $c, h$}

The strategy of the proof will be quite simple. Beginning with a quasiautomorphic function we associate a differential equation as in the previous section. Using any automorphism $\varphi$ we conjugate the differential equation. The new differential equation defines a new quasiautomorphic function which is simply related to the old one. This relation is impossible unless the functions are in fact the same, which implies that the exponents of $q$ are fixed by all automorphisms of $\mathbf{C}$ and hence rational. Let us now spell this out in more detail.

First we define the notion of conjugation by an algebraic automorphism. If $f$ is any function on the upper half plane with a $q$-Puiseux expansion

$$
f=\sum a_{i} q^{\omega_{i}}
$$

we define $f^{\varphi}$ to be the function (if it exists) whose $q$-Puiseux expansion is obtained by acting with $\varphi$ on the coefficients and exponents of $q$ :

$$
f^{\varphi}=\sum \varphi\left(a_{i}\right) q^{\varphi\left(\omega_{i}\right)} .
$$

If $f$ is a rational function of $\lambda$ then it is of the form

$$
f=\frac{\sum r_{i} \lambda^{i}}{\sum s_{i} \lambda^{i}}
$$

(both sums finite). Since the $q$ (-Puiseux) expansion of $\lambda$ has integral coefficients, and since $\varphi$ is an automorphism, we see that in fact

$$
f^{\varphi}=\frac{\sum \varphi\left(r_{i}\right) \lambda^{i}}{\sum \varphi\left(s_{i}\right) \lambda^{i}} .
$$

Thus, the $\varphi$-conjugate of a rational function of $\lambda$ exists and is another rational function of $\lambda$.

We use this observation in the proof of the following. 
Proposition 2. Let $\varphi$ be an algebraic automorphism of $\mathbf{C}$ and let

$$
f=\sum a_{n} q^{\omega_{n}}
$$

be quasiautomorphic, then

$$
f^{\varphi}=\sum \varphi\left(a_{n}\right) q^{\varphi\left(\omega_{n}\right)}
$$

exists and is quasiautomorphic.

Proof. By Proposition 1, Span $\{f \circ \gamma\}_{\gamma \in \Gamma(2)}$ is precisely the set of solutions of a differential equation

$$
\left(\frac{d}{d \lambda}\right)^{n} y+k_{1}\left(\frac{d}{d \lambda}\right)^{n-1} y+\cdots+k_{n} y=0
$$

enjoying the properties $\left(\mathrm{A}^{\prime}\right)$. If we conjugate the coefficients $k_{i}$ of (3.1) we obtain a differential equation

$$
\left(\frac{d}{d \lambda}\right)^{n} y+k_{1}^{\varphi}\left(\frac{d}{d \lambda}\right)^{n-1} y+\cdots+k_{n}^{\varphi} y=0,
$$

which again possesses the properties $\left(\mathrm{A}^{\prime}\right)$. The solutions of (3.2) may be obtainedafter a certain amount of transformation - by the method of Frobenius. (For details see [15].) Thus the formal solutions of (3.2) are the same as the true solutions, and since $f^{\varphi}$ formally satisfies (3.2) it must be the $q$-Puiseux expansion of a genuine solution of (3.2). Therefore, $f^{\varphi}$ exists and is a quasiautomorphic function by Proposition 1. This proves Proposition 2.

Let us now reconsider the setting of the introduction. We observe that the operator $T: \tau \rightarrow \tau+1$ in the quasiautomorphic representation $\operatorname{Span}\left\{f_{i}\right\}$ which arises in rational conformal field theory is diagonalizable. In order to see this, note first that any basis $\vec{f}$ transforms as

$$
T \vec{f}=e^{2 \pi i \theta} \vec{f}
$$

where $\theta$ is a matrix. Then, the vector

$$
\vec{g}=e^{-2 \pi i \theta \tau} \vec{f}
$$

is a vector of periodic functions, which therefore have $q$-expansions. Assuming, as we may, that $\theta$ is in Jordan canonical form then, by (1.4) $\vec{f}$ has a $q$-Puiseux expansion; since $\vec{g}$ has a $q$-expansion the Jordan blocks of $\theta$ are one-by-one, hence $\theta$ is diagonal, i.e. $T$ is diagonalizable. Thus we can choose our basis $\left\{f_{i}\right\}$ so that

$$
f_{i}=\sum_{n=0}^{\infty} d_{n i} q^{n+\theta_{r}}
$$

Moreover we can choose $d_{n i} \in \mathbf{Z}$.

We are finally ready to use Proposition 2 to establish.

Proposition 3. With $f_{i}$ as above, all the exponents $\theta_{i}$ are rational.

Proof. Let $f$ stand for any of the $f_{i}$ above. By Proposition 2, $g=f^{\varphi}$ is 
quasiautomorphic, but $g=e^{\alpha \tau} f$ with $\alpha=2 \pi i(\varphi(\theta)-\theta)$. Applying the transformation $\tau \rightarrow-1 / \tau$ to this equation we have

$$
\tilde{g}=e^{-\alpha / \tau} \tilde{f}
$$

where $\tilde{g}, \tilde{f}$ are quasiautomorphic functions in the span of $g, f$. Since the basis vectors $f_{i}$ have $q$-Puiseux expansions, it follows that the elements in the representation generated by $g$ likewise have $q$-Puiseux expansions, hence $\tilde{f}, \tilde{g}$ can be written

$$
\tilde{g}=\sum s_{i} q^{\varepsilon_{i}}, \quad \tilde{f}=\sum t_{i} q^{\rho_{i}} .
$$

Suppose that one exponent $\xi \in\left\{\varepsilon_{i}, \rho_{i}\right\}$ has the property that $\operatorname{Re}(\xi)$ is strictly smaller than the real parts of all other exponents. Then, write:

$$
\frac{\tilde{g}-\tilde{f}}{q^{\xi}}=\left(\frac{e^{-\alpha / \tau}-1}{q^{\xi}}\right) \tilde{f} .
$$

As $\tau \rightarrow i \infty$ along the imaginary axis the left-hand side of this equation has a finite nonzero limit whereas the right-hand side has a limit which is either zero or infinity, a contradiction.

If there are several $\xi$ which minimize $\operatorname{Re}(\xi)$, then replacing the limit $\tau \rightarrow i \infty$ along the imaginary axis with the limit $\tau \rightarrow e^{(\pi / 2+\varepsilon) i} \infty$ along a ray at a angle $\varepsilon$ to the imaginary axis, then, one term will again dominate and the above argument again leads to contradiction. Thus, it must be that $\varphi(\theta)=\theta$ for all algebraic automorphisms of $\mathbf{C}$. This implies $\theta \in \mathbf{Q}$, thus concluding the argument.

From Proposition 3 and the previous remarks we obtain the theorem stated in the introduction, and hence the rationality of $c, h$ in rational conformal field theory.

\section{Conclusions}

There is a conjecture that the value of $c$ in conformal field theory is always rational. The corresponding statement is certainly not true for $h$. Unfortunately, the methods of this paper probably cannot be extended to the general case: the obstruction is that algebraic automorphisms are highly discontinuous. It is possible that these ideas can be extended to theories in which the values of $h$ lie in a finite algebraic extension of $\mathbf{Q}$. Examples of such conformal field theories are known [21].

The fact that the expansion coefficients of the quasiautomorphic functions appearing in conformal field theories are nonnegative integers is an extremely strong constraint. It is possible that $p$-adic analysis can be used to deduce other constraints on the data $h, c$ following purely from (1.4) and (1.3). For example, it is conceivable that in rational conformal field theories the denominators of $c, h$ are related to the dimension of the vector bundle over moduli space. Another interesting question is whether the quasiautomorphic functions appearing in rational conformal field theory are always algebraic functions of $\lambda$. No examples of RCFT are known in which this is not the case. 
The work of G. A. was supported in part by NSF grant DMS-8610730(2); The work of G. M. was supported by DOE contract DE-AC02-76ER-02220. G. A. also thanks the Sloan foundation for support.

\section{References}

1. Belavin, A., Polyakov, A., Zamolodchikov, A.: Infinite conformal symmetry in two-dimensional quantum field theory. Nucl. Phys. B241, 333 (1984)

2. Cardy, J.: Operator content of two-dimensional conformally invariant theories. Nucl. Phys. B270, $186(1986)$

3. Friedan, D., Shenker, S.: Lectures at IAS, 1987

4. Moore, G.: Nucl. Phys. B293, 139 (1987)

5. Kastor, D., Martinec, E., Qiu, Z.: Current algebra and conformal discrete series. Chicago preprint EFI-87-58

6. Dijkgraaf, R., Verlinde, E., Verlinde, H.: Conformal field theory at $c=1$. Utrecht preprints THU-87/17; THU-87/27

7. Friedan, D., Qiu, Z., Shenker, S. H.: Conformal invariance unitarity, and critical exponents in two dimensions. Phys. Rev. Lett. 52, 1575 (1984)

8. Zamalodchikoc, A. B., Fateev, V. A.: Nonlocal (parafermion) currents in two-dimensional conformal quantum field theory and self-dual critical points in $z_{N}$-symmetric statistical systems. JETP $\mathbf{6 2}$, Disorder fields in two-dimensional conformal quantum-field theory and $N=z$ extended supersymmetry. JETP 63, 913 (1986)

9. Gepner, D., Qiu., Z:: Modular invariant partition functions for parafermionic field theories. Nucl. Phys. B285, 423 (1987)

10. Bagger, J., Nemeschansky, D., Yankielowicz, S.: Virasoro algebras with central charge $c>1$. Harvard preprint HUTP-87/A073

11. See, e.g. Gepner, D., Witten, E.: String theory on group manifolds. Nucl. Phys. B278, 493 (1986); Bernard, D., Thierry-Mieg, J.: Bosonic Kac-Moody string theories. Phys. Lett. 185B 65, (1986) and references therein

12. Ginsparg, P.: Curiosities at $c=1$ Harvard preprint. HUTP-87/A068

13. Friedan, D., Shenker, S.: The integrable analytic geometry of quantum string. Phys. Lett. 175B, 287 (1986); The analytic geometry of two-dimensional conformal field theory. Nucl. Phys. B281, 509 (1987)

14. Ahlfors, L.: Complex analysis. New York: McGraw Hill 1966. See the last chapter

15. Deligne, P.: Equations différentielles à points singuliers réguliers. Lecture Notes in Mathematics, Vol. 163, Berlin, Heidelberg, New York: Springer 1970

16. Katz, N.: Publ. IHES, 39, 355

17. Eguchi, T., Ooguri, H.: Differential equations for conformal characters in moduli space. Tokyo preprint

18. Kazhdan, D., Vafa, C.: unpublished

19. Herstein, I. N.: Topics in algebra Xerox, 1975

20. Lang, S.: Algebra. Reading, MA: Addison Wesley 1971

21. Harvey, J., Moore, G., Vafa, C.: Quasicrystalline compactification. PUPT-1068; IADDNS-HEP87/48; HUTP-87/A072

Communicated by L. Alvarez-Gaumé

Received December 22, 1987 